\title{
Multi-Pattern Output Consensus in Networks of Heterogeneous Nonlinear Agents with Uncertain Leader: a Nonlinear Regression Approach
}

\author{
Giacomo Casadei and Daniele Astolfi
}

\begin{abstract}
In this paper we consider the problem of consensus of a network of heterogeneous nonlinear agents on a family of different desired trajectories generated by an uncertain leader. We design a set of local reference generators and local controllers which guarantees that the agents achieve consensus robustly on all possible trajectories inside this family. The design of the local reference generators is based on the possibility to express the trajectory of the leader as a nonlinear regression law which is parametrized by some constant unknown parameters.
\end{abstract}

Index Terms-Synchronization and consensus, uncertain nonlinear systems, nonlinear regression.

\section{INTRODUCTION}

The problem of consensus and synchronization has attracted a lot of attention from the control community. Often, multi-agent systems and networks of different nature exhibit agreements as one of their main feature. This type of agreement can be modeled by means of consensus/synchronization behavior and control techniques are designed in order to steer the agents to achieve such a coordination. The typical field of application of these concepts is the control of multi-agent robotic systems, where the agents have to find a common trajectory to coordinate their motion (see [1], [2]). However, many other domains, such as power networks (see [3]) and social networks (see [4]), have pushed the researchers to deepen into the properties of networks.

From a theoretical point of view, the problem of consensus and synchronization in networks of homogeneous linear and nonlinear systems has been studied by several authors and seminal results can be found in [5]-[8]. Subsequently, the challenging problem of networks of heterogeneous systems as been studied and many authors have considered various aspects of such a problem (between the others, see [9] for the linear case, [10] for nonlinear case). In [11] it has been proved that a necessary and sufficient condition for output synchronization in heterogeneous networks is the internal model principle. This concept has been adapted and extended to the nonlinear case in [12] and has become the widely accepted technique to face the problem known in literature as cooperative output regulation. The key aspect of this

G. Casadei is with Univ. Grenoble Alpes, CNRS, Inria, GIPSA-lab, France, giacomo.casadiedgipsa-lab. fr. This project has received funding from the European Research Council (ERC) under the European Union's Horizon 2020 research and innovation programme (grant agreement $\mathrm{N}^{\circ} 694209$ ).

D. Astolfi is with the Université de Lorraine, CRAN, UMR 7039, Nancy, France and the CRNS, CRAN, UMR 7039, Nancy, France, daniele.astolfieuniv-lorraine.fr approach is that the consensus trajectory can be thought as generated by an exosystem, whose model is known to all the agents in the network. This exosystems, replicated in each agent's controller and synchronized with the others agents', provides a reference trajectory to be tracked locally. As a matter of fact, the knowledge a priori of this reference trajectory is in many cases a really strong assumption. One may think to the case in which we want a network of followers to synchronize on a leader's trajectory, which is unknown or just partially-known to the followers.

Several authors have focused on uncertain networks: on one hand, these uncertainties might involve the agents themselves and not the exosystem (see for instance [13], [14]): this problem however reduces to a local robustness issue. On the other hand, the problem of uncertain exosystems has been taken into account for instance in [15], where the authors consider a neural network approach to estimate the unknown parameters, yet achieving only practical synchronization. In [16], the author treats the case of a multi-pattern linear exosystem: synchronization is achieved by regulation theory, while the particular trajectory depends on the initial conditions of the agents. In [17]-[19], the authors exploit adaptive architecture to estimate the unknown parameters of the linear leader and synchronize the agents to the generated reference.

In this paper, we consider a network of nonlinear heterogeneous agents which have to track a nonlinear reference generated by a nonlinear leader, whose trajectory is not known a priori. In contrast with conventional adaptive techniques, we show that an opportune design of a set of local reference generators, based on a nonlinear regression law, guarantees robust synchronization on the leader reference trajectory.

\section{Communication Graphs}

The exchange of information between the agents is described by a directed time-invariant graph. A graph can be described by a triplet $\mathcal{G}=\{\mathcal{V}, \mathcal{E}, A\}$ in which:

- $\mathcal{V}$ is a set of $N$ nodes $\mathcal{V}=\left\{v_{1}, v_{2}, \ldots, v_{N}\right\}$, one for each of the $N$ agents in the set;

- $\mathcal{E} \subset \mathcal{V} \times \mathcal{V}$ is a set of edges that models the interconnection between nodes, according to the following convention: $\left(v_{k}, v_{j}\right)$ belongs to $\mathcal{E}$ if there is a flow of information from node $j$ to node $k$;

- the flow of information from node $j$ to node $k$ is weighted by the $(k, j)$-th entry $a_{k j} \geq 0$ of the adjacency matrix $A \in \mathbb{R}^{N \times N}$. 
By definition, the Laplacian matrix is defined as

$$
\ell_{k j}=-a_{k j} \quad \text { if } \quad k \neq j, \quad \ell_{k j}=\sum_{i=1}^{N} a_{k i} \quad \text { if } \quad k=j .
$$

The diagonal entries of $L$ are non-negative, the off-diagonal entries are non-positive and, for each row, the sum of all entries on this row is zero. A matrix with these properties is usually referred to as a Metzler matrix. As a consequence, the all-ones $N$-vector $1_{N}=\operatorname{col}(1,1, \ldots, 1)$ is an eigenvector of $L$, associated with the eigenvalue $\lambda=0$. Let the other (possibly nontrivial) $N-1$ eigenvalues of $L$ be denoted as $\lambda_{2}(L), \ldots, \lambda_{N}(L)$.

Theorem 1 A time-invariant graph is connected if and only if $L$ has only one trivial eigenvalue $\lambda_{1}=0$ and all other eigenvalues $\lambda_{2}(L), \ldots, \lambda_{N}(L)$ have positive real parts.

\section{Problem Formulation}

In this paper, we consider a network of $N$ nonlinear agents with a leader. We assume the graph is leader-connected, namely there exists a direct path from the leader to all the other agents.

Assumption 1 The graph $\mathcal{G}$ is leader-connected, namely there exists a path from the leader to all other nodes. Thus, there exists a positive scalar $\alpha$ such that $\alpha \leq \Re\left(\lambda_{i}(L)\right)$ for $i=2, \ldots, N$.

Without loss of generality, the leader-node is labeled as the first agent: hence, the Laplacian matrix takes the form

$$
L=\left[\begin{array}{c}
\mathbf{0}_{1 \times N} \\
\tilde{L}_{N-1 \times N}
\end{array}\right] \text {. }
$$

The information available for control purpose at the $k$-th agent depends on the set of neighbors of agent $k$ and can be expressed according to Laplacian matrix $L$ as

$$
v_{k}=-\sum_{j=1}^{N} \ell_{k j} \sigma_{j} \quad k=1, \ldots, N
$$

with $\sigma_{j}$ the selected output of agent $j$, for $j=1, \ldots, N$.

\section{A. Description of the Agents}

The unknown leader trajectory $y^{\star}(t)$ determines the pattern on which the followers are asked to synchronize on. This trajectory is not known a priori and it depends on a set of constant parameters $\mu$ ranging in some compact set $\mathcal{U} \subset \mathbb{R}^{n_{\mu}}$. Different values of the unknown parameters $\mu$ may cause different reference trajectories, motivating the definition of multi-pattern consensus. In this paper we focus on the class of consensus bounded trajectories $y^{\star}(t)$ that can be generated by a nonlinear autonomous system fulfilling a regression formula of order $d$ which is linear in the parameters $\mu$, namely ${ }^{1}$

$$
y^{\star(d)}=\mathcal{H}\left(y_{[0, d-1]}^{\star}\right)+\mathcal{F}\left(y_{[0, d-1]}^{\star}\right) \mu
$$

\footnotetext{
${ }^{1}$ Here and in the following, $f_{[a, b]}$ stands for the the vector of derivatives of the function $f(\cdot)$ from order $a$ to order $b$, namely $f_{[a, b]}=$ $\left(f^{(a)}, \ldots, f^{(b)}\right)^{T}$.
}

where $\mathcal{H}: \mathbb{R}^{d} \rightarrow \mathbb{R}$ and $\mathcal{F}: \mathbb{R}^{d} \rightarrow \mathbb{R}^{n_{\mu}}$ are functions smooth enough. Alternatively, the leader's dynamics can be expressed as a dynamical system of dimension $d$ of the form

$$
\begin{aligned}
& \dot{x}_{1}=S x_{1}+B\left(\mathcal{H}\left(x_{1}\right)+\mathcal{F}\left(x_{1}\right) \mu\right), \quad x_{1} \in \mathbb{R}^{d}, \\
& y^{\star}=C x_{1},
\end{aligned}
$$

with initial conditions $x_{1}(0)$ ranging in some given compact set $X^{\star} \subset \mathbb{R}^{d}$ and where $(S, B, C)$ is a triplet of matrices in prime form ${ }^{2}$ of dimension $d$. In particular, we ask system (4) to fulfil the following assumption (we refer to [20] for the notion of Poisson stability).

Assumption 2 Let $\mu$ belong to a compact set $\mathcal{U} \subset \mathbb{R}^{n_{\mu}}$. The system (4) is Poisson stable "uniformly in $\mu$ ", namely for any $\mu \in \mathcal{U}$ there exists a compact invariant set $X^{\star}(\mu) \subset \mathbb{R}^{d}$ for (4). We denote with $X^{\star}$ the union of all $X^{\star}(\mu)$.

The remaining $N-1$ heterogeneous nonlinear agents are described by

$$
\begin{aligned}
\dot{x}_{k} & =f_{k}\left(x_{k}, u_{k}\right), \\
y_{k} & =h_{k}\left(x_{k}\right),
\end{aligned} \quad x_{k} \in \mathbb{R}^{n_{k}}, u_{k}, y_{k} \in \mathbb{R},
$$

for $k=2, \ldots, N$, where $u_{k}$ and $y_{k}$ are the local control input and output. Our goal is to define $u_{k}$ such that the $N-1$ followers achieve consensus on the trajectory $y^{\star}$ generated by the unknown leader. Each agent controller takes the form of

$$
\begin{aligned}
& \dot{\eta}_{k}=\Phi_{k}\left(\eta_{k}, y_{k}, v_{k}\right), \quad \eta_{k} \in \mathbb{R}^{\bar{n}_{k}}, v_{k} \in \mathbb{R}^{p}, \\
& u_{k}=\Gamma_{k}\left(\eta_{k}, y_{k}, v_{k}\right), \\
& \sigma_{k}=\Sigma_{k}\left(\eta_{k}, y_{k}\right), \quad \sigma_{k} \in \mathbb{R}^{p},
\end{aligned}
$$

in which $v_{k}$ and $\sigma_{k}$ are respectively inputs and outputs that characterize the exchange of information between individual agents. Then the control problem can be defined in the following way. Let $X_{k} \subset \mathbb{R}^{n_{k}}$, for $k=2, \ldots, N$, be the compact set of admissible initial condition for each follower (5). The goal is to design $N-1$ controller (6), with set of initial condition $H_{k}$, such that, for any $\left(x_{k}(0), \eta_{k}(0)\right) \in X_{k} \times H_{k}$ and for all $k=2, \ldots, N$, we have

$$
\lim _{t \rightarrow \infty} y^{\star}(t)-y_{k}(t)=0 \text {. }
$$

\section{B. Structure of Local Controllers}

By following [11]-[12], the local controllers (6) can be designed in two steps. By defining $\eta_{k}$ in (6) as $\eta_{k}=$ $\operatorname{col}\left(w_{k}, \zeta_{k}\right)$, the local controllers can be written as

$$
\begin{aligned}
\dot{w}_{k} & =s\left(w_{k}\right)+K v_{k} \\
\dot{\zeta}_{k} & =\varphi_{k}\left(\zeta_{k}, y_{k}, w_{k}\right) \\
u_{k} & =\gamma_{k}\left(\zeta_{k}, y_{k}, w_{k}\right) \\
\sigma_{k} & =c\left(w_{k}\right)
\end{aligned}
$$

where $v_{k}$ is defined according to (2) and the matrix $K$ and the functions $s(\cdot), \varphi_{k}(\cdot), \gamma_{k}(\cdot)$ and $c(\cdot)$ have to be designed.

${ }^{2}$ A triplet of matrices $(S, B, C)$ is said to be in prime form when $S$ is a shift matrix (all 1's on the upper diagonal and all 0's elsewhere), $B^{T}=$ $\left(\begin{array}{lll}0 & \cdots & 0\end{array}\right)$ and $C=\left(\begin{array}{lll}1 & 0 & \cdots\end{array}\right)$. 
The $w_{k}$-dynamics can be seen as a set of local reference generators(LRG)

$$
\dot{w}_{k}=s\left(w_{k}\right)+K v_{k}, \quad y_{k}^{\text {ref }}=c\left(w_{k}\right),
$$

coupled to the other agents' LRG via

$$
v_{k}=-\sum_{j=1}^{N} \ell_{k j} c\left(w_{j}\right),
$$

each one providing a local reference $y_{k}^{\text {ref }}$ to be tracked. By defining the local tracking error $e_{k}$ as

$$
e_{k}:=y_{k}-y_{k}^{\text {ref }} \text {, }
$$

the $\zeta_{k}$-dynamics represent the set of $N-1$ local regulators

$$
\begin{aligned}
\dot{\zeta}_{k} & =\varphi_{k}\left(\zeta_{k}, y^{\text {ref }}+e_{k}, w_{k}\right) \\
u_{k} & =\gamma_{k}\left(\eta_{k}, y^{\text {ref }}+e_{k}, w_{k}\right)
\end{aligned}
$$

designed in such a way that $\lim _{t \rightarrow \infty} e_{k}(t)=0$. The design of (7) is divided in two steps. First, we will design (8) in such a way that local reference $y_{k}^{\text {ref }}$ converges to the leader's reference $y^{\star}$. Then, we will define (11) to steer the local tracking error (10) to zero, which in turn guarantees output consensus of the heterogeneous network.

\section{SyNCHRONIZATION OF THE LOCAL REFERENCE GENERATORS}

In view of the control architecture introduced in Section II-B, the main difficulty resides in the design of the LRG (8) to cope with the unknown leader's parameters $\mu \in \mathbb{R}^{n_{\mu}}$. In contrast with adaptive solutions, we propose a robust technique which was originally presented in [21] and applied to the framework of output regulation in [22]. To this end, by differentiating $i$ times (3), we obtain

$$
y_{[d, d+i]}^{\star}=\mathcal{H}_{i}\left(y_{[0, d+i-1]}^{\star}\right)+\mathcal{F}_{i}\left(y_{[0, d+i-1]}^{\star}\right) \mu,
$$

where

$$
\begin{aligned}
& \mathcal{H}_{i}\left(y_{[0, d+i-1]}^{\star}\right)=\operatorname{col}\left(\mathcal{H}_{0}\left(y_{[0, d-1]}^{\star}\right), \ldots \mathcal{H}_{i}\left(y_{[0, d+i-1]}^{\star}\right)\right), \\
& \mathcal{F}_{i}\left(y_{[0, d+i-1]}^{\star}\right)=\operatorname{col}\left(\mathcal{F}_{0}\left(y_{[0, d-1]}^{\star}\right), \ldots, \mathcal{F}_{i}\left(y_{[0, d+i-1]}^{\star}\right)\right),
\end{aligned}
$$

with $\mathcal{H}_{0}(\cdot)=\mathcal{H}(\cdot), \mathcal{F}_{0}(\cdot)=\mathcal{F}(\cdot)$ and $\mathcal{H}_{j}(\cdot)=\dot{\mathcal{H}}_{j-1}(\cdot)$ and $\mathcal{F}_{j}(\cdot)=\dot{\mathcal{F}}_{j-1}(\cdot)$ for $j=1, \ldots, i$. If there exists a number $m \geq n_{\mu}$ such that the matrix $\mathcal{F}_{m}(\cdot)$ is pseudo-invertible, then it is possible to replace $\mu$ in (12) with

$$
\mu=\mathcal{F}_{m}^{+}\left(y_{[0, d+m-1]}^{\star}\right)\left(y_{[d, d+m]}^{\star}-\mathcal{H}_{m}\left(y_{[0, d+m-1]}^{\star}\right)\right)
$$

where $\mathcal{F}_{m}^{+}(\cdot)$ denotes the pseudo-inverse of $\mathcal{F}_{m}(\cdot)$. We thus obtain a new formulation equivalent to (12) of order $\bar{d}=d+$ $m+1$. Existence of the pseudo-inverse of the matrix $\mathcal{F}_{m}(\cdot)$ is guaranteed under the following persistence of excitation condition.

Assumption 3 There exist $m \geq n_{\mu}$ and $\delta>0$ such that

$$
\operatorname{det}\left(\mathcal{F}_{m}^{T}\left(y_{[0, d+m-1]}^{\star}\right) \mathcal{F}_{m}\left(y_{[0, d+m-1]}^{\star}\right)\right) \geq \delta
$$

for any trajectory $y^{\star}(t)$ generated by the system (4), under Assumption 2, with $\mu \in \mathcal{U}$ and $x \in X^{\star}$.
Persistence of excitation assumptions are recurrent in presence of unknown parameters. In this paper we do not adopt any well known adaptive techniques that already have been considered in the case of networks, e.g. [17]-[19]. Instead, we will exploit this assumption showing that it corresponds to an observability condition of the unknown parameters, thus allowing to achieve asymptotic synchronization without measuring directly $\mu$. In particular, as shown in Proposition 2 in [22], under Assumption 3, the reference signal (3) can be thought as generated by an autonomous dynamical system of dimension $\bar{d}$, namely

$$
\begin{aligned}
\dot{w}_{1} & =S w_{1}+B \phi\left(w_{1}\right), \quad w_{1} \in \mathbb{R}^{\bar{d}}, \\
y^{\star} & =C w_{1},
\end{aligned}
$$

where the initial conditions $w_{1}(0)$ range in some compact set $W^{\star} \subset \mathbb{R}^{\bar{d}}$ to be specified later, the matrices $(S, B, C)$ are a triplet in prime form of dimension $\bar{d}$, and the function $\phi(\cdot)$ is defined in particular as

$\phi\left(w_{1}\right):=\mathcal{H}_{m+1}\left(w_{1}\right)+\mathcal{F}_{m+1}\left(w_{1}\right) \mathcal{F}_{m}^{+}\left(w_{1}\right)\left(\mathcal{T} w_{1}-\mathcal{H}_{m}\left(w_{1}\right)\right)$,

with $\mathcal{T}:=\left(\begin{array}{ll}0_{(m+1) \times d} & I_{(m+1) \times(m+1)}\end{array}\right)_{(m+1) \times(d+m+1)}$. As shown in [22], Assumption 3 and smoothness of the functions $\mathcal{H}(\cdot)$ and $\mathcal{F}(\cdot)$ implies that the function $\phi(\cdot)$ is at least locally Lipschitz. Having in mind the definition of the system (4), we define the compact invariant set $W^{\star} \subset \mathbb{R}^{\bar{d}}$ such that, for any trajectory $x_{1}(t)$ generated by the dynamical system (4) with parameters $\mu \in \mathcal{U}$ and evolving in the compact space $X^{\star}$, there exists a corresponding trajectory $w_{1}(t)$ of (14) evolving in $W^{\star}$ such that the two outputs $y^{\star}\left(x_{1}\right)$ and $y^{\star}\left(w_{1}\right)$ coincides.

In particular, the set $W^{\star}$ can be constructed as follows. Pick any $\mu \in \mathcal{U}$ and let $W_{i}^{\star}(\mu)$ denote a compact subset of $\mathbb{R}$. For $i=1, \ldots, d$ we chose $W_{i}^{\star}(\mu)$ such that

$$
X^{\star}(\mu)=W_{1}^{\star}(\mu) \times \cdots \times W_{d}^{\star}(\mu) .
$$

Then, for $i>d$, we

$$
\begin{array}{r}
W_{d+j}^{\star}(\mu):=\left\{r \in \mathbb{R}: r=\mathcal{H}_{j-1}(z)+\mathcal{F}_{j-1}(z) \mu,\right. \\
\left.\forall z \in \prod_{k=1}^{d+j-1} W_{k}^{\star}(\mu)\right\},
\end{array}
$$

with $j=1, \ldots, m+1$. Finally, we construct $W^{\star}(\mu)$ as

$$
W^{\star}(\mu):=W_{1}^{\star}(\mu) \times \cdots \times W_{d+m+1}^{\star}(\mu) .
$$

Lastly, we select $W^{\star}$ as the union of all the $W^{\star}(\mu)$ for any $\mu \in \mathcal{U}$. As a consequence it can be verified by construction that for any initial condition $x_{1}(0) \in X^{\star}$ and any parameter $\mu \in \mathcal{U}$, there exists a corresponding initial condition $w_{1}(0) \in W^{\star}$ such that the trajectories $x_{1}(t)$ and $w_{1}(t)$ respectively generated by the systems (4) and (14) satisfy $w_{1_{i}}(t)=x_{1_{i}}(t)=y^{\star(i-1)}(t)$ for all $t \geq 0$ and for all $i=1, \ldots, d$, where $x_{1_{i}}$ denotes the $i$-th component of $x_{1}$ and $w_{i_{1}}$ denotes the $i$-th component of $w_{1}$. In other words, instead of considering the reference trajectory $y^{\star}(t)$ and satisfying (3) as generated by $x_{1}$ in (4), whose dimension is $d$, with $\mu \in \mathcal{U}$ viewed as an (unknown) input, we consider 
$y^{\star}(t)$ as generated by an autonomous dynamical systems $w_{1}$ expressed by (14). Systems (4) and (14) are thus equivalent representations of the reference trajectory $y^{\star}(t)$ at hand. In order to better explain the previous procedure we provide two examples where the formulation (14) can be used to cope with parametric uncertainties of the leader trajectory and multi-pattern leader trajectories. Other examples can be found in [21].

\section{A. Examples}

1) Synchronization with Parametric Uncertainties: Consider the case in which the leader trajectory $y^{\star}$ is generated by a linear oscillator with unknown frequency of oscillation $\omega$, namely

$$
\ddot{y}^{\star}+\omega^{2} y^{\star}=0 .
$$

Evidently the reference trajectory (15) can be written in the form (4) or alternatively as

$$
\dot{x}_{1}=x_{2}, \quad \dot{x}_{2}=-x_{3} x_{1}, \quad \dot{x}_{3}=0, \quad y=x_{1}
$$

where $x_{3}=\omega^{2}$ represents the unknown parameter. By defining $w_{1}=\left(w_{11}, w_{12}, w_{13}, w_{14}\right)^{T} \in \mathbb{R}^{4}$ according to

$$
w_{11}=x_{1}, \quad w_{12}=x_{2}, \quad w_{13}=-x_{3} x_{1}, \quad w_{14}=-x_{3} x_{2},
$$

we obtain a system in the form (14) with $\bar{d}=4$, where

$$
\phi(w)=\left(\frac{w_{11} w_{13}+w_{12} w_{14}}{w_{11}^{2}+w_{12}^{2}}\right)^{2} w_{11} .
$$

It is readily seen that, by following the previous procedure, it is possible to cope with the more general case of trajectories generated by uncertain linear systems of the form

$$
\dot{v}=S(\mu) v, \quad y^{\star}=P v
$$

with $S(\mu)$ being a neutrally stable matrix for any $\mu \in \mathcal{U}$. This case has been already addressed in literature but with adaptive techniques. See, for instance, [17]-[19].

2) Robust multi-pattern synchronization: As mentioned before, the procedure presented above can be seen as a robust synchronization tool in case of leader's multi-pattern trajectories. Consider for instance the case in which the leader might generate three different trajectories uncertain in the parameters, namely an uncertain Van Der Pool, an uncertain Duffing oscillator and an uncertain linear oscillator. We consider therefore a $y^{\star}$ satisfying

$$
\ddot{y}^{\star}=\alpha y^{\star}+\beta \dot{y}^{\star}+\gamma y^{\star 3}+\delta y^{\star 2} \dot{y}^{\star},
$$

which, for different configurations of the parameters $\alpha, \beta, \gamma, \delta$, includes the three aforementioned oscillators (and actually many others). It is trivially seen that we can express (16) according to (12) in the compact form

$$
\ddot{y}^{\star}=\mathcal{F}\left(y_{[0,1]}^{\star}\right) \mu,
$$

where $\mathcal{F}\left(x_{[0,1]}\right)=\left(y^{\star}, \dot{y}^{\star}, y^{\star 3}, y^{\star 2} \dot{y}^{\star}\right)$ and $\mu=$ $\operatorname{col}(\alpha, \beta, \gamma, \delta)$. By differentiating (17) to the 4-th order, we obtain $y_{[2,6]}^{\star}=\mathcal{F}_{4}\left(y_{[0,5]}^{\star}\right) \mu$. It is possible to check numerically (see [22]) that the matrix $\mathcal{F}_{4}(\cdot) \in \mathbb{R}^{4 \times 5}$ is pseudo-invertible when the trajectory of $y^{\star}$ is generated as the first component of a Van De Pool oscillator, a Duffing oscillator or a linear oscillator. Thus the parameters $\mu$ can be computed according to (13). This in turn allows to embed the multi-pattern uncertain trajectory (16) in a system of the kind of (14) of order $\bar{d}=7$.

\section{B. Design of the Local Reference Generator}

Having in mind the description (14) of the leader dynamics, the LRGs (8) are designed by selecting the function $s(\cdot)$ and $c(\cdot)$ as

$$
s\left(w_{k}\right)=S w_{k}+B \bar{\phi}\left(w_{k}\right), \quad c\left(w_{k}\right)=C w_{k}
$$

with $(S, B, C)$ a triplet in prime form of dimension $\bar{d}$, initial conditions $w_{k}(0)$ ranging in any compact set $W_{k} \subset \mathbb{R}^{\bar{d}}$ and where the functions $\bar{\phi}(\cdot)$ is any bounded locally Lipschitz function that agrees with $\phi(\cdot)$ on the set $W^{\star}$. We denote with $\mathcal{L}>0$ the real number satisfying

$$
\left|\bar{\phi}\left(w_{k}^{a}\right)-\phi\left(w_{k}^{b}\right)\right| \leq \mathcal{L}\left|w_{k}^{a}-w_{k}^{b}\right|,
$$

for any $\left(w_{k}^{a}, w_{k}^{b}\right) \in \mathbb{R}^{\bar{d}} \times W^{\star}$. From a practical point of view, it is interesting to remark that we do not have to compute "formally" the function $\mathcal{F}_{m}^{+}(\cdot)$ in order to implement the function $\phi(\cdot)$. Rather, it can be evaluated on-line since it requires only the computation of the pseudo-inverse of a matrix. Finally, note that the existence of the function $\bar{\phi}(\cdot)$ is guaranteed by the Tietze Extension Theorem. It can be selected, for instance, by saturating the function $\phi(\cdot)$ outside the compact set $W^{\star}$.

Next, we define $K$ as

$$
K=D_{g} K_{0},
$$

where $D_{g} \in \mathbb{R}^{\bar{d} \times \bar{d}}$ is defined as $D_{g}=\operatorname{diag}\left(g, \ldots, g^{\bar{d}}\right)$ with $g \geq 1$ the so called "high-gain parameter" (see [20]) and $K_{0}=P C^{T}$ with $P$ solution of the Riccati equation

$$
S P+P S^{T}-\alpha P C^{T} C P=-a I,
$$

where $a \in \mathbb{R}_{>0}$ is a free design parameter and $\alpha$ is given by Assumption 1. The forthcoming proposition states that with the previous design of the functions $s(\cdot), c(\cdot)$ and $K$, asymptotic synchronization of the local reference generators (8) with the leader (4) is achieved robustly with respect to the parameters $\mu$ ranging in the compact set $\mathcal{U}$.

Proposition 1 Suppose Assumptions 1, 2 and 3 hold. Choose the functions $s(\cdot), c(\cdot)$ according to (18) and the matrix $K$ according to (20). Then there exists $g^{\star} \geq 1$ such that for any $g \geq g^{\star}$, for any initial condition $w_{k}(0) \in W_{k} \subset \mathbb{R}^{d}$, with $k=2, \ldots, N, x_{1}(0) \in X_{1}$ and for any constant parameter $\mu \in \mathcal{U}$, the trajectories of (8), coupled via (9), are bounded for all $t \geq 0$ and satisfy $\lim _{t \rightarrow \infty} e_{k}(t)=0$, for all $k=$ $2, \ldots, N$, with $e_{k}$ defined in (10).

Proof: For dimensional consistency, we consider the leader trajectory $y^{\star}$ as generated by the system (14). Then, 
by letting $\mathbf{w}=\operatorname{col}\left(w_{1}, w_{2}, \ldots, w_{N}\right)$, the network of (8)-(14) can be written in compact form as

$$
\dot{\mathbf{w}}=\left(I_{N} \otimes S\right) \mathbf{w}+\left(I_{N} \otimes B\right) \Phi(\mathbf{w})+(L \otimes K C) \mathbf{w}
$$

where $\Phi(\mathbf{w})=\operatorname{col}\left(\phi\left(w_{1}\right), \bar{\phi}\left(w_{w}\right), \ldots, \bar{\phi}\left(w_{N}\right)\right)$. The result can be proved by showing that the set

$$
\mathcal{W}=\left\{\mathbf{w} \in W^{\star} \times \ldots \times W^{\star}: w_{1}=\ldots=w_{N}\right\}
$$

is asymptotically stable for (22), semi-globally with respect to $w_{k}$ for $k=2, \ldots, N$. To do so, let $T \in \mathbb{R}^{N \times N}$ be such that

$$
\tilde{L}=T^{-1} L T=\left[\begin{array}{cc}
0 & \mathbf{0}_{1 \times N-1} \\
\mathbf{0}_{N-1 \times 1} & L_{22}
\end{array}\right],
$$

with eig $\left(L_{22}\right)=\left\{\lambda_{2}(L), \ldots, \lambda_{N}(L)\right\}$. We change coordinate according to $\mathbf{w} \mapsto \mathbf{z}:=\left(T^{-1} \otimes I_{\bar{d}}\right) \mathbf{w}$, which component wise reads as

$$
\begin{aligned}
w_{1} & \mapsto z_{1}:=w_{1}, \\
w_{k} & \mapsto z_{k}:=w_{k}-w_{1}, \quad k=2, \ldots, N .
\end{aligned}
$$

In the new coordinates, system (22) reads

$$
\begin{aligned}
& \dot{z}_{1}=S z_{1}+B \phi\left(z_{1}\right) \\
& \dot{\mathbf{z}}_{2}=\boldsymbol{A}\left(z_{1}, \mathbf{z}_{\mathbf{2}}\right)
\end{aligned}
$$

where $\mathbf{z}_{2}=\operatorname{col}\left(z_{2}, \ldots, z_{N}\right)$ and

$\boldsymbol{A}(\mathbf{z}):=\left[\left(I_{N} \otimes S\right)-\left(L_{22} \otimes K C\right)\right] \mathbf{z}_{2}+\left(I_{N} \otimes B\right) \Delta \Phi\left(z_{1}, \mathbf{z}_{2}\right)$,

where the $i$-component of $\Delta \Phi(\cdot, \cdot): \mathbb{R}^{(N-1) \bar{d}} \rightarrow \mathbb{R}^{(N-1)}$ is given by $\bar{\phi}\left(z_{i}-z_{1}\right)-\phi\left(z_{1}\right)$. Note that, by definition, $\mathbf{z}_{2}=0$ implies $\bar{\phi}\left(z_{1}\right)=\phi\left(z_{1}\right)$ and thus $\Delta \Phi\left(z_{1}, 0\right)=0$, uniformly in $z_{1}$. Since the subsystem (24) is autonomous, in the rest of the proof, we will focus on proving asymptotic stability of the origin of $\mathbf{z}_{2}$-dynamics (25), which in turn, in view of (23), implies that synchronization is achieved. To do so, we change coordinate according to

$$
\mathbf{z}_{2} \mapsto \vartheta:=\left(T_{J}^{-1} \otimes D_{g}^{-1}\right) \mathbf{z}_{2},
$$

with $T_{J}$ such that $L_{J}=T_{J}^{-1} L_{22} T_{J}$ is the Jordan canonical form of $L_{22}$. For the sake of simplicity, in the following we suppose that $L_{J}$ has a purely diagonal form (the not-diagonal case follows trivially, by means of ISS arguments). In the new coordinates, system (25) reads as

$$
\dot{\boldsymbol{\vartheta}}=g \boldsymbol{\Lambda} \boldsymbol{\vartheta}+\frac{1}{g^{\bar{d}}} \Delta\left(z_{1}, \boldsymbol{\vartheta}\right) .
$$

where $\boldsymbol{\Lambda}=\left[\left(I_{N} \otimes S\right)+\left(L_{J} \otimes K_{0} C\right)\right]=$ blkdiag $\left(\Lambda_{2}, \ldots, \Lambda_{N}\right)$ with $\Lambda_{i}=S-\lambda_{i}(L) K_{0} C$ for $k=$ $2, \ldots, N$, and $\Delta\left(z_{1}, \boldsymbol{\vartheta}\right):=\left(T_{J}^{-1} \otimes B\right) \Delta \Phi\left(z_{1},\left(T_{J} \otimes D_{g}\right) \boldsymbol{\vartheta}\right)$, is such that $\Delta\left(z_{1}, 0\right)=0$, uniformly in $z_{1}$. Consider now the Lyapunov function $V=\boldsymbol{\vartheta}^{T}\left(I_{N-1} \otimes P^{-1}\right) \boldsymbol{\vartheta}$, and recall that by definition of $P$ in (21), we have $P^{-1} \Lambda_{i}+\Lambda_{i}^{-1} P^{-1}=-a I$. As a consequence, by differentiating with respect to time we obtain

$$
\begin{aligned}
\dot{V} & =-a g\|\boldsymbol{\vartheta}\|^{2}+g^{-\bar{d}} \boldsymbol{\vartheta}^{T}\left(I_{N-1} \otimes P^{-1}\right) \Delta\left(z_{1}, \boldsymbol{\vartheta}\right) \\
& \leq-a g\|\boldsymbol{\vartheta}\|^{2}+\beta \mathcal{L}\|\boldsymbol{\vartheta}\|^{2} .
\end{aligned}
$$

where, by definition of $\Delta(\cdot)$ and (19), $\beta>0$ is such that

$$
\left\|g^{-\bar{d}} \boldsymbol{\vartheta}^{T}\left(I_{N-1} \otimes P^{-1}\right) \Delta\left(z_{1}, \boldsymbol{\vartheta}\right)\right\| \leq \beta \mathcal{L}\|\boldsymbol{\vartheta}\|^{2},
$$

for all $\vartheta \in \mathbb{R}^{(N-1) \bar{d}}$ and $z_{1} \in W^{\star}$. As a consequence, the previous inequality leads to

$$
\dot{V} \leq-a g\|\boldsymbol{\vartheta}\|^{2}+\beta \mathcal{L}\|\boldsymbol{\vartheta}\|^{2} .
$$

By selecting any $g^{\star}>\max \{\beta \mathcal{L} / a, 1\}$ and by combining the definition of $V$ and the change of coordinates (26) it comes straightforward the existence of real numbers $b_{1}>0, b_{2}>0$ (independent of $g$ ) such that the following holds

$$
\left\|\mathbf{z}_{2}(t)\right\| \leq b_{1} g^{\bar{d}} \exp \left(-b_{2} g t\right)\left\|\mathbf{z}_{2}(0)\right\|, \quad \forall t \geq 0,
$$

for any $g \geq g^{\star}$. By definition of $\mathbf{z}_{2}=\operatorname{col}\left(z_{2}, \ldots, z_{N}\right)$ in (23), we conclude that the followers' local reference generators synchronize on the leader's trajectory.

It is worth noticing that the gain $D_{g}$ implemented in (20) is composed by increasing powers of $g$ up to $g^{\bar{d}}$. As a consequence, when $\bar{d}$ or $g$ are very large, the local reference generators (18) can be designed as in [22] by means of the novel "low-power" technique introduced for high-gain observers in [23] to avoid numerical implementation issues.

\section{Local Tracking Problem and}

\section{SYNCHRONIZATION OF THE OUTPUT OF THE AGENTS}

In Section III, we showed that synchronization of the followers' LRG $w_{k}$ on the leader trajectory $w_{1}$ can be obtained by an opportune design of the dynamics (8) and of the control parameter $K$ according to (18) and (20). By recalling the definition $e_{k}$ in (10), Proposition 1 states that $\lim _{t \rightarrow \infty} y_{k}(t)=y^{\star}(t)$ for all $k=2, \ldots, N$. As a consequence, we are left with a set of $N-1$ local tracking problems which in turn involves the design of $N-1$ local controller such that each agent (5) tracks its local reference trajectory generated by (8). In the following assumption, we ask that there exists a dynamic regulator (11), such that each agent (5) is capable of robustly tracking the leader's reference trajectory generated by (14).

Assumption 4 Consider, for any $k=2, \ldots, N$, the system

$$
\begin{array}{ll}
\dot{w}_{1}=S w_{1}+B \phi\left(w_{1}\right), & y^{\star}=C w_{1}, \\
\dot{x}_{k}=f_{k}\left(x_{k}, u_{k}\right), & y_{k}=h_{k}\left(x_{k}\right),
\end{array}
$$

with initial conditions $\left(w_{1}(0), x_{k}(0)\right)$ in $W^{\star} \times X_{k}$. Let $\epsilon_{k}>0$ be any (small) positive real number. There exist functions $\hat{\varphi}_{k}(\cdot)$ and $\hat{\gamma}_{k}(\cdot)$, such that the system (28) in closed-loop with the dynamic regulator

$$
\begin{aligned}
\dot{\zeta}_{k} & =\hat{\varphi}_{k}\left(\zeta_{k}, y_{k}, w_{1}+\varepsilon(t)\right) \\
u_{k} & =\hat{\gamma}_{k}\left(\eta_{k}, y_{k}, w_{1}+\varepsilon(t)\right)
\end{aligned}
$$

with initial condition $\zeta_{k}(0) \in Z_{k}$, satisfies the following:

i) there exists a compact set $W^{\star} \times \bar{X}_{k}$, with $\bar{X}_{k} \supset X_{k}$, which is forward invariant for any $\varepsilon \in L_{l o c}^{\infty}\left(\mathbb{R}, \mathbb{R}^{\bar{d}}\right)$ satisfying $\|\varepsilon(t)\| \leq \epsilon_{k}$ for all $t \geq 0$;

ii) the compact set $\mathcal{B}=\left\{\left(w_{1}, x_{k}\right) \in W^{\star} \times \mathbb{R}^{n_{k}}: y_{k}-y^{\star}=\right.$ $0\}$ is asymptotically stable with a domain of attraction $\mathcal{A} \supset W^{\star} \times \bar{X}_{k}$ for $\varepsilon(t)=0$. 

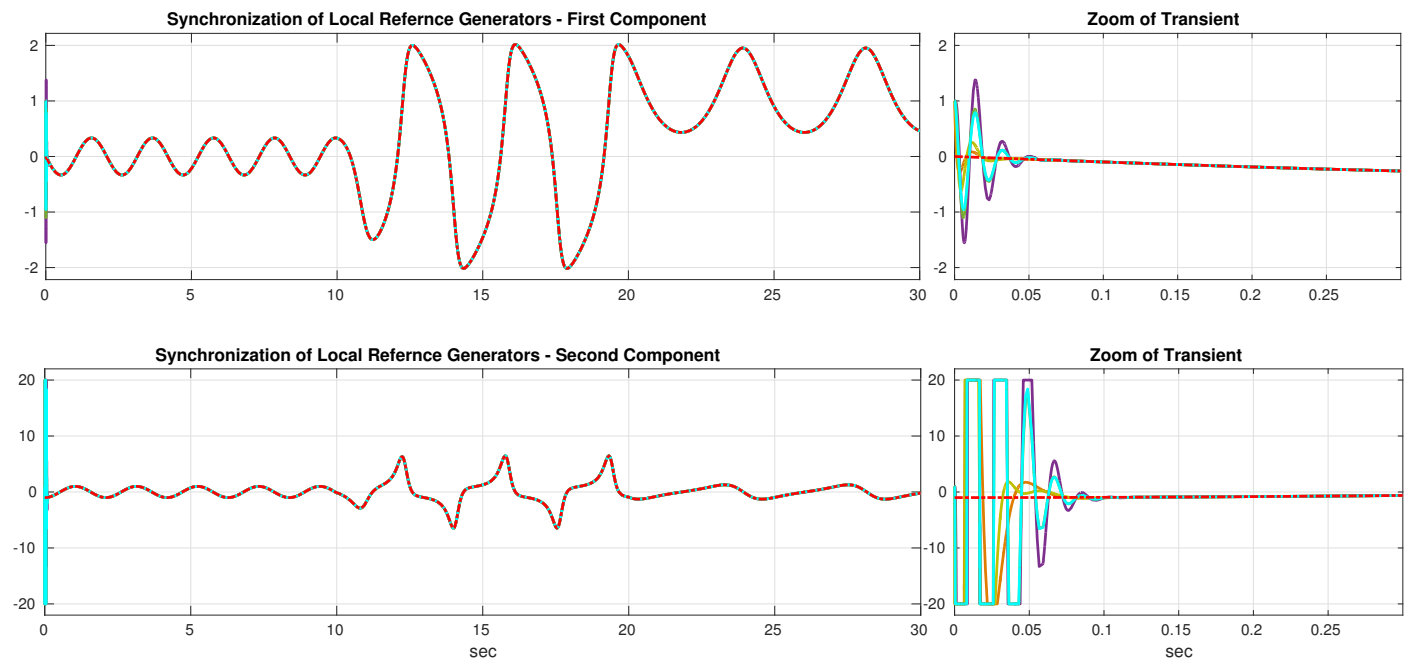

Fig. 1. Synchronization of the local reference generators $y_{k}^{\text {ref }}$ on the leader's trajectory. The leader's trajectory is plotted as a dashed-dotted red line: during the transient, the local reference generators saturates according to the chosen values and ultimately synchronize on the leader's trajectory.

In our framework, the $N-1$ agents (5) do not have access to the leader's reference trajectory and its derivatives but only to the set of local reference generator (8). During the transient, the state of the system (8) may leave the compact set $W^{\star}$. As a consequence, the controller $\hat{\varphi}_{k}, \hat{\gamma}_{k}$ in Assumption 4 is modified as follows

$$
\begin{aligned}
\varphi_{k}\left(\zeta_{k}, y_{k}, w_{k}\right) & :=\hat{\varphi}_{k}\left(\zeta_{k}, y_{k}, \mathcal{I}_{k}\left(w_{k}\right)\right), \\
\gamma_{k}\left(\eta_{k}, y_{k}, w_{k}\right) & :=\hat{\gamma}_{k}\left(\eta_{k}, y_{k}, \mathcal{I}_{k}\left(w_{k}\right)\right),
\end{aligned}
$$

where $\mathcal{I}_{k}(\cdot)$ is any continuous bounded function that agrees with the identity function in the set $W^{\star}$ and satisfying

$$
\left|\mathcal{I}_{k}\left(w_{k}\right)\right| \leq \sup _{w_{k} \in W^{\star}}\left|w_{k}\right|+\epsilon_{k} \quad \forall w_{k} \in \mathbb{R}^{\bar{d}} .
$$

The next proposition summarizes the main result of the paper, namely the output synchronization of the $N-1$ nonlinear agents (5) with the leader trajectory (4).

Proposition 2 Let Assumptions 1-4 hold. Then, for every initial condition $\left(w_{k}, x_{k}, \zeta_{k}\right) \in W_{k} \times X_{k} \times Z_{k}$, the solution of the system (5) in closed-loop with the controller (7) and with the leader trajectory $y^{\star}$ generated by (3), is bounded and satisfies $\lim _{t \rightarrow \infty} y_{k}(t)-y^{\star}(t)=0$ for all $k=2, \ldots, N$.

Proof: The proof is a direct result of Proposition 1 and Assumption 4. In particular, Proposition 1 guarantees that the $\boldsymbol{\vartheta}$-dynamics are 0-GAS and consequently the $\mathbf{z}_{2}$-dynamics. The latter can be shown by recalling the definition of $z_{k}$ and $\mathbf{z}_{2}$ in (23) and (25) and the fact that $z_{1}=w_{1}$. With this in mind, it is possible to write the dynamics of the close-loop system (3)-(5)-(7) in the compact form

$$
\begin{aligned}
\dot{w}_{1} & =S w_{1}+B \phi\left(w_{1}\right), & \dot{z}_{k} & =A_{k}\left(w_{1}, \mathbf{z}_{2}\right), \\
\dot{\zeta}_{k} & =\varphi\left(\zeta_{k}, y_{k}, w_{1}+\nu_{k}\right), & \dot{x}_{k} & =f_{k}\left(x_{k}, u_{k}\right),
\end{aligned}
$$

where $A_{k}(\cdot)$ denotes the $k$-th row of the function $\boldsymbol{A}(\cdot)$ in (25) and $\nu_{k}$ is defined as $\nu_{k}:=\mathcal{I}_{k}\left(z_{k}+w_{1}\right)-w_{1}$. Note that by definition of the function $\mathcal{I}_{\epsilon}(\cdot)$, we have $\left|\nu_{k}(t)\right| \leq$ $\epsilon$ for all $t \geq 0$. As a consequence, by using the result of
Proposition 1 and item $i$ ) in Assumption 4, we have that $x_{k}(t) \in \bar{X}_{k}$ for $t \geq 0$. By recalling (27) and the definition of $\mathbf{z}_{2}=\operatorname{col}\left(z_{2}, \ldots, z_{N}\right)$, we obtain $\lim _{t \rightarrow \infty} z_{k}(t)=0$ and therefore $\lim _{t \rightarrow \infty} \nu_{k}(t)=0$, for any $k=2, \ldots, N$, The proof concludes by using item $i i$ ) of Assumption 4 .

We conclude this section by stressing that in order to design the dynamic regulator (29) one can use any tracking/regulation technique satisfying Assumption 4. This choice may depend in general on the properties of system (5). For instance, under a minimum phase assumption, one may use nonlinear output regulation tools [20], as shown in [12]. Moreover, since in our framework each agent has the information of the tracking reference $y_{k}^{\text {ref }}$ and its $\bar{d}$ derivatives, it is possible to exploit this knowledge to use other (feedforward) tracking techniques, such as feedback linearization, [24], flatness approaches, [25] sliding mode control, [26], or neural network approaches, [15].

\section{Simulations}

To confirm the results presented in Section III and Section IV, we consider the case of 5 nonlinear heterogeneous agents which are asked to synchronize on a leader trajectory whose dynamics is described by (16). In the simulations, the leader node describes a linear oscillator $(\alpha=9, \beta=\gamma=\delta=0)$ in the time interval $[0,10] \mathrm{sec}$, then smoothly changes into a Van Der Pool oscillator $(\alpha=-1, \beta=3, \gamma=0$, $\delta=3)$ in the time interval $[10,20] \mathrm{sec}$, and finally into a Duffing oscillator $(\alpha=-1, \beta=0, \gamma=-2, \delta=0)$ in the time interval $[20,30] \mathrm{sec}$. Following the example presented in Section III-A2, the leader dynamics can be thought as generated by a system of the form of (14) with $\bar{d}=7$. Then, the local reference generator is designed according to (18) with $K$ defined as in (20)-(21) with $a=1$ and $g=400$. The final reference trajectory is generated by designing the function $\mathcal{I}_{k}\left(w_{k}\right)$ as a component-wise saturation with values $(10,20,70,200,500,3500,40000)$, for all $k$. The 5 agents are 


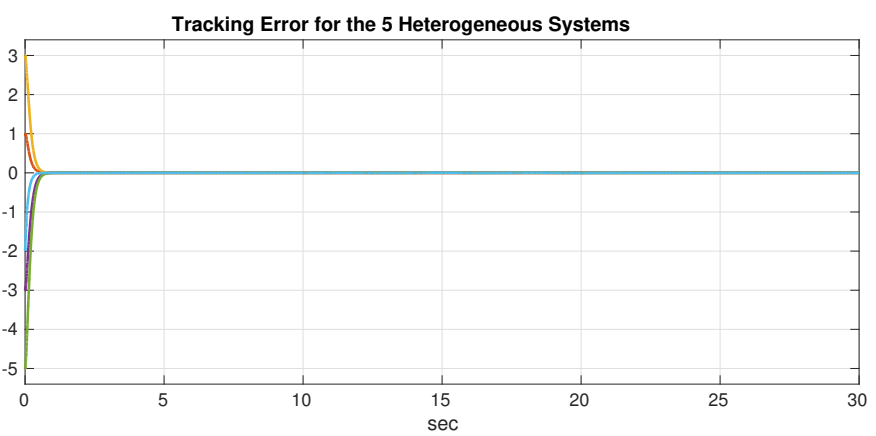

Fig. 2. Tracking error of the 5 followers with respect to the reference trajectory.

heterogeneous both in the dynamics and in the dimension, namely $x_{i} \in \mathbb{R}^{2}$ for $i=2,3,4$ and $x_{i} \in \mathbb{R}^{3}$ for $i=5,6$,

$\left\{\begin{array}{l}\dot{x}_{2_{1}}=x_{2_{2}} \\ \dot{x}_{2_{2}}=\frac{-x_{2_{1}}}{1+x_{2_{1}}^{2}}+u_{2} \\ y_{2}=x_{2_{1}}\end{array}\left\{\begin{array}{l}\dot{x}_{3_{1}}=x_{3_{2}} \\ \dot{x}_{3_{2}}=-x_{3_{1}}+u_{3} \\ y_{3}=x_{3_{1}}\end{array}\left\{\begin{array}{l}\dot{x}_{4_{1}}=x_{4_{2}}-x_{4_{1}} \\ \dot{x}_{4_{2}}=x_{4_{1}}^{2}+u_{4} \\ y_{4}=x_{4_{1}}\end{array}\right.\right.\right.$
$\left\{\begin{array}{l}\dot{x}_{5_{1}}=x_{5_{2}}+x_{5_{3}} \\ \dot{x}_{5_{2}}=x_{5_{1}} x_{5_{2}} \\ \dot{x}_{5_{3}}=-x_{5_{1}}+u_{5} \\ y_{5}=x_{5_{1}}\end{array} \quad\left\{\begin{array}{l}\dot{x}_{6_{1}}=28\left(x_{6_{3}}-x_{6_{1}}\right) \\ \dot{x}_{6_{2}}=x_{6_{1}} x_{6_{3}}-\frac{8}{3} x_{6_{2}} \\ \dot{x}_{6_{3}}=10 x_{6_{1}}-x_{6_{3}}-x_{6_{3}} x_{6_{2}}^{2}+u_{6} \\ y_{6}=x_{6_{1}}\end{array}\right.\right.$

Each regulator (11) is designed with an observer to reconstruct the state $x_{k}$ from the output $y_{k}$ and a nonlinear stabilizer to steer the tracking error to zero. The particular design of the regulators are omitted for the sake of compactness. Figure 1 shows the synchronization of the local reference generators $y_{k}^{\mathrm{ref}}$ on the leader's trajectory: even if the leader changes its trajectory from linear to nonlinear dynamics, the network of followers is capable of tracking all the different trajectories. Figure 2 shows the tracking error for the 5 heterogeneous agents with respect to the reference trajectory.

\section{CONCLUSION}

In this paper we considered the problem of synchronization of a network of heterogeneous nonlinear agents with unknown leader. By exploiting nonlinear regression tools, we designed a set of local reference generators capable of robustly synchronizing over the unknown leader's trajectory. Then, by solving a set of local tracking problems, we guaranteed that the heterogeneous agents outputs synchronize with the leader. From a theoretical point of view, this technique can be extended by considering the case of switching communication networks, for instance by following the approach introduced in [27]. On the application side, it would be interesting to consider its application to electrical power networks where the power load variation could be monitored according to the proposed approach, e.g. [28].

\section{REFERENCES}

[1] R. Olfati-Saber, "Flocking for multi-agent dynamic systems: Algorithms and theory", IEEE Trans. Aut. Contr., 51(3), pp. 401-420, 2006.

[2] R. Sepulchre, D. A. Paley, and N. E. Leonard, "Stabilization of planar collective motion with limited communication", IEEE Trans. Aut. Control, 53(3), pp. 706-719, 2008.
[3] S. V. Dhople, B. B. Johnson, F. Dörfler, A. O. Hamadeh, "Synchronization of nonlinear circuits in dynamic electrical networks with general topologies", IEEE Transactions on Circuits and Systems-I: Regular Papers, 61(9), pp. 2677-2690, 2014.

[4] A. Mirtabatabaei, and F. Bullo, "Opinion dynamics in heterogeneous networks: Convergence conjectures and theorems", SIAM Journal on Contr. and Optim., 50(5), pp. 2763-2785, 2012.

[5] L. Moreau, "Stability of continuous-time distributed consensus algorithms", Proc. CDC, pp. 3998-4003, 2004.

[6] J. Qin, H. Gao and W. X. Zheng, "Exponential Synchronization of Complex Networks of Linear Systems and Nonlinear Oscillators: A Unified Analysis", IEEE TNNLS, 26(3), pp. 510-521, 2015.

[7] M. Arcak, "Passivity as a design tool for group coordination", IEEE IEEE Trans. Aut. Control, vol. 52(8), pp. 1380-1390, 2007.

[8] G.B. Stan and R. Sepulchre, "Analysis of interconnected oscillators by dissipativity theory", IEEE Trans. Aut. Control, vol. 52(2), pp. 256270, 2007.

[9] G. Seyboth, D. Dimarogonas, K. H. Johansson, P. Frasca, F. Allgöwer, "On robust synchronization of heterogeneous linear multi-agent systems with static couplings", Automatica, vol. 53, pp. 392-399, 2015.

[10] Z. Ding, "Consensus Output Regulation of a Class of Heterogeneous Nonlinear Systems", IEEE Trans. Autom. Control, vol. 58(10), pp. 2648-2653, 2013

[11] P. Wieland, R.Sepulchre, F. Allgöwer, "An internal model principle is necessary and sufficient for linear output synchronization", Automatica, vol. 47, pp. 1068-1074, 2011.

[12] A. Isidori, L. Marconi, G. Casadei, "Robust Output Synchronization of a Network of Heterogeneous Nonlinear Agents Via Nonlinear Regulation Theory", IEEE Trans. Aut. Control, vol. 59(10), pp. 2680 2692, 2014.

[13] H. Kim, H. Shim, and J.H. Seo. "Output consensus of heterogeneous uncertain linear multi-agent systems", IEEE Trans. Aut. Control, vol. 56(1), pp. 200-206, 2011.

[14] H. Liu, C. De Persis, M. Cao, "Robust decentralized output regulation with single or multiple reference signals for uncertain heterogeneous systems", Int. Journal of Robust and Nonlinear Control, vol. 25(9), pp. 1399-1422, 2014

[15] Z. Peng, D. Wang, H. Zhang and G. Sun, "Distributed Neural Network Control for Adaptive Synchronization of Uncertain Dynamical Multiagent Systems", IEEE Trans. Neur. Netwroks Learn. Sys., vol. 25(8), pp. 1508-1519, 2014

[16] Z. Chen, "Pattern Synchronization of Nonlinear Heterogeneous Multiagent Networks With Jointly Connected Topologies", IEEE Trans. Contr. Netwrok Sys., vol. 1(4), pp. 349-359, 2014.

[17] Y. Su and J. Huang, "Cooperative adaptive output regulation for a class of nonlinear uncertain multi-agent systems with unknown leader", System \& Control Letters, vol. 62, pp. 461-467, 2013.

[18] X. Wang, Y. Hong, H. Ji, "Adaptive multi-agent containment control with multiple parametric uncertain leaders", Automatica, vol. 50(9), pp. 2366-2372, 2014.

[19] Y. Dong, "Rendezvous with connectivity preservation for multi-robot systems with an unknown leader", Int. Journ. of Contr., 2017.

[20] C.I. Byrnes and A. Isidori. "Nonlinear Internal Models for Output Regulation", IEEE Trans. Aut. Control, vol. 49(12), pp. 2244-2247, 2004.

[21] F. Forte, A. Isidori, L. Marconi. "Robust design of internal models by nonlinear regression", 9th IFAC Symposium on Nonlinear Control Systems, vol. 46(23), pp. 283-288, 2013.

[22] M. Bin, D. Astolfi, L. Marconi. "Robust Internal Model Design by Nonlinear Regression via Low-Power High-Gain Observers", 55th IEEE Conf. on Dec. and Contr., 2016.

[23] D. Astolfi, L. Marconi. "A High-Gain Nonlinear Observer with Limited Gain Power", IEEE Trans. Aut. Control, vol. 60(11), pp. 3059-3064, 2015.

[24] H. K. Khalil, "Nonlinear Control", Pearson, 2015.

[25] J. Lévine, "Analysis and Control of Nonlinear Systems, a Flatnessbased Approach", Springer, 2009.

[26] Y. Shtessel, C. Edwards, L. Fridman and A. Levant, "Sliding Mode Control and Observation", Springer, 2014

[27] G. Casadei, L. Marconi, A. Isidori, "About disconnected topologies and synchronization of homogeneous nonlinear agents over switching networks", DOI: 10.1002/rnc.3910, 2017.

[28] M. Bürger, C. De Persis, "Dynamic coupling design for nonlinear output agreement and time-varying flow control", Automatica, 51, pp. 210-222, 2015 